\title{
On a question of Beardon
}

\section{Guowei Zhang*}

${ }^{\text {*Correspondence: }}$ zhirobo@gmail.com

School of Mathematics and

Statistics, Anyang Normal University,

Anyang, 455000, P.R. China

\begin{abstract}
In this paper, we estimate the order of growth of the solutions of the equation $f(k z)=k f(z) f^{\prime}(z)$ and investigate the periodicity of the solutions in the case $k=3$, which give an answer to the question proposed by Beardon (Comput. Methods Funct. Theory 12(1):273-278, 2012).
\end{abstract}

MSC: 30D35; 30D45

Keywords: complex difference; Nevanlinna theory; growth

\section{Introduction and main results}

As entire functions $z, \sin z, \sinh z$ are solutions of the equation $f(2 z)=2 f(z) f^{\prime}(z)$, Beardon [1] studied entire solutions of the generalized functional equation

$$
f(k z)=k f(z) f^{\prime}(z), \quad f(0)=0,
$$

where $k$ is a non-zero complex number. Obviously, two formal power series $f$ and $g$ are linearly conjugate if there is a non-zero $c$ such that $g(x)=c f(x / c)$, and if $f$ satisfies (1.1).

Firstly, we define some notations as in the paper [1]. The formal series $\mathcal{O}$ and $\mathcal{I}$ are defined by $\mathcal{O}:=0+0 z+0 z^{2}+\cdots, \mathcal{I}:=0+1 z+0 z^{2}+0 z^{3}+\cdots$. We also introduce sets $\mathcal{K}_{p}=\left\{z: z^{p}=p+2\right\}(p=1,2, \ldots)$ and $\mathcal{K}=\mathcal{K}_{1} \cup \mathcal{K}_{2} \cup \ldots$. Thus, we have $\mathcal{K}_{1}=3$ and $\mathcal{K}_{2}=$ $\{-2,2\}$. Obviously, $\mathcal{K}_{p}$ contains exactly $p$ points which are equally spaced around the circle $|z|=R_{p}$, where $R_{p}=(p+2)^{1 / p}>1$ and $R_{p} \in \mathcal{K}_{p}$. Also, since $x^{-1} \log (x+2)$ is decreasing when $x>1$, we see that $R_{1}=3>R_{2}=2>\cdots>1$, and $R_{p} \rightarrow 1$ as $p \rightarrow \infty$. In particular, the sets $\mathcal{K}_{p}$ are mutually disjoint, and the derived set of $\mathcal{K}$ is the unit circle $\{z:|z|=1\}$. Using the above notations, Beardon obtained the following two main results for the entire solutions of equation (1.1).

Theorem A Any transcendental solution $f$ of (1.1) is of the form

$$
f(z)=z+z\left(b z^{p}+\cdots\right)
$$

where $p$ is a positive integer, $b \neq 0$ and $k \in \mathcal{K}_{p}$. In particular, if $k \notin \mathcal{K}$ then the only formal solutions of (1.1) are $\mathcal{O}$ and $\mathcal{I}$.

Theorem B For each positive integer $p$, there is a unique real entire function

$$
F_{p}(z)=z\left(1+z^{p}+b_{2} z^{2 p}+b_{3} z^{3 p}+\cdots\right)
$$

\section{Springer}

(0) 2013 Zhang; licensee Springer. This is an Open Access article distributed under the terms of the Creative Commons Attribution License (http://creativecommons.org/licenses/by/2.0), which permits unrestricted use, distribution, and reproduction in any medium, provided the original work is properly cited. 
which is a solution of (1.1) for each $k$ in $\mathcal{K}_{p}$. Further, if $k \in \mathcal{K}_{p}$ then the only transcendental solutions of (1.1) are the linear conjugates of $F_{p}$.

Based on the above two known results, we use the value distribution theory in $q$-difference (see, e.g., [2-6]), which is analogue of the classical Nevanlinna theory of meromorphic functions (see, e.g., [7-9]), to study the properties of solutions of (1.1). We get the upper bound of the order of solutions (see [10]).

Theorem 1 Suppose that $f$ is a transcendental solution of (1.1) for $k \in \mathcal{K}$, then the order $\lambda(f) \leq \frac{\log 2}{\log |k|}$.

In particular, when $k=3$, the order of solutions of $f(3 z)=3 f(z) f^{\prime}(z)$ is not more than $\log 2 / \log 3$. In Section 3 of the paper [1], Beardon also studied the periodicity of the solutions of equation (1.1). Although the solutions of (1.1) are periodic when $k= \pm 2$ (that is, $p=2$ ), he proved that the periodicity fails when $p \geq 3$, see [1, Theorem 3.1]. But the case $p=1$ (that is, $k=3$ ) remains open. Here we shall prove that the periodicity also fails for the remaining case.

Theorem 2 The solution $f$ of equation (1.1) is not periodic when $k=3$.

From Theorem 1, we know that the order of the transcendental solution $f$ is not more than 1 when $k=2$. This coincides with the fact that the transcendental solutions are $\sin z$ and $\sinh z$, the order of which are 1 . Naturally we ask: Is the order of transcendental solutions of equation (1.1) exactly $\log 2 / \log |k|$ ? That means we have to estimate the lower bound of the order of solutions. Unfortunately, we do not get the expected lower bound since we meet difficulties when using $T\left(r, f^{\prime}\right)$ to bound $T(r, f)$, because for any given positive constant $K$, there exists an entire function $f$ with order $\lambda$ for which

$$
\frac{T(r, f)}{T\left(r, f^{\prime}\right)}>K
$$

on a set $E$ of positive lower logarithmic density; see Hayman [11, p.98]. So the above question is open.

\section{Some lemmas}

In this paper we use the standard notations in the Nevanlinna theory (see, e.g., [7-9]). So, in the following we give some well-known results, which are needed for our proof, of the classical Nevanlinna theory without presenting proofs. Let $f(z)$ be a meromorphic function, and let $m(r, f), N(r, f), T(r, f)$ denote the proximity function, the counting function and characteristic function of $f(z)$, respectively, here $r=|z| . T(r, f)=m(r, f)+N(r, f)$ and for the entire function $N(r, f)=0$. Moreover, the order of growth of a meromorphic function $f(z)$ is defined by

$$
\lambda(f):=\limsup _{r \rightarrow \infty} \frac{\log T(r, f)}{\log r} .
$$

We denote by $E$ a set of finite linear measure in $R^{+}$, not necessarily the same at each occurrence. For any non-constant meromorphic function $f(z)$, we denote by $S(r, f)$ any quantity 
satisfying $S(r, f)=o(T(r, f))(r \rightarrow \infty, r \notin E)$. For two meromorphic functions $f(z)$ and $g(z)$, we have $m(r, f g) \leq m(r, f)+m(r, g)$ and $T(r, f g) \leq T(r, f)+T(r, g)$. In addition, the identity $m\left(r, \frac{f^{\prime}}{f}\right)=S(r, f)$ is also a very important result in the Nevanlinna theory.

The first lemma on the relationship between $T(r, f(q z))$ and $T(|q| r, f(z))$ is due to Bergweiler et al. [12, p.2].

Lemma 2.1 One case, see that

$$
T(r, f(q z))=T(|q| r, f)+O(1)
$$

holds for any meromorphic function $f$ and any constant $q$.

Lemma 2.2 [13] Let $\Phi:(1, \infty) \rightarrow(0, \infty)$ be a monotone increasing function, and let $f$ be a nonconstant meromorphic function. If for some real constant $\alpha \in(0,1)$, there exist real constants $K_{1}>0$ and $K_{2} \geq 1$ such that

$$
T(r, f) \leq K_{1} \Phi(\alpha r)+K_{2} T(\alpha r, f)+S(\alpha r, f),
$$

then the order of growth of $f$ satisfies

$$
\lambda(f) \leq \frac{\log K_{2}}{-\log \alpha}+\limsup _{r \rightarrow \infty} \frac{\log \Phi(r)}{\log r}
$$

Lemma 2.3 [9, Lemma 5.1] Suppose that a nonconstant meromorphic function $f$ is periodic, that is, $f(z+\eta)=f(z)$ for nonzero complex number $\eta$. Then the order $\lambda(f) \geq 1$.

\section{Proof of theorems}

Proof of Theorem 1 By the definition of $\mathcal{K}$, we know that $|k|>1$. Thus, by Lemma 2.1 we have

$$
T(r, f(k z))=T(|k| r, f(z))+O(1),
$$

and by (1.1), we can get

$$
T(r, f(k z))=T\left(r, k f(z) f^{\prime}(z)\right) \leq T(r, f(z))+T\left(r, f^{\prime}(z)\right)+O(1) .
$$

Combining the two inequalities above and simplifying $T(r, f(z))$ by $T(r, f)$, we have

$$
T(|k| r, f) \leq T(r, f)+T\left(r, f^{\prime}\right)+O(1) .
$$

By Theorem B, we know that the solution $f$ is entire. Since for the entire function $f$ its derivative is also entire, we have

$$
\begin{aligned}
T\left(r, f^{\prime}\right) & =m\left(r, f^{\prime}\right)=m\left(r, f^{f^{\prime}}\right) \leq m(r, f)+m\left(r, \frac{f^{\prime}}{f}\right) \\
& =m(r, f)+S(r, f)=T(r, f)+S(r, f) .
\end{aligned}
$$


By (3.3) and (3.4), we have

$$
T(|k| r, f) \leq 2 T(r, f)+S(r, f) .
$$

Set $\alpha=1 /|k|$, thus, we get

$$
T(r, f) \leq 2 T(\alpha r, f)+S(\alpha r, f) .
$$

Applying Lemma 2.2 yields

$$
\lambda(f) \leq \frac{\log 2}{\log |k|} .
$$

Proof of Theorem 2 Theorem 2 follows from Theorem 1 and Lemma 2.3.

\section{Competing interests}

The author declares that he has no competing interests.

\section{Acknowledgements}

The author wishes to express his thanks to the referee for his/her valuable suggestions and comments. The present investigation was supported by the National Natural Science Foundation under Grant No. 11226088 and the Key Project of Natural Science Foundation of Educational Committee of Henan Province under Grant No. 12A110002 of the People's Republic of China.

Received: 18 April 2013 Accepted: 8 July 2013 Published: 22 July 2013

\section{References}

1. Beardon, AF: Entire solutions of $f(k z)=k f(z) f^{\prime}(z)$. Comput. Methods Funct. Theory 12(1), 273-278 (2012)

2. Barnett, DC, Halburd, RG, Korhonen, RJ, Morgan, W: Nevanlinna theory for the $q$-difference operator and meromorphic solutions of q-difference equations. Proc. R. Soc. Edinb. A 137, 457-474 (2007)

3. Chiang, YM, Feng, SJ: On the Nevanlinna characteristic of $f(z+\eta)$ and difference equations in the complex plane. Ramanujan J. 16, 105-129(2008)

4. Chiang, YM, Feng, SJ: On the growth of logarithmic differences, difference quotients and logarithmic derivatives of meromorphic functions. Trans. Am. Math. Soc. 361(7), 3767-3791 (2009)

5. Laine, I, Yang, CC: Clunie theorems for difference and q-difference polynomials. J. Lond. Math. Soc. 2(76), 556-566 (2007)

6. Laine, I, Yang, CC: Value distribution of difference polynomials. Proc. Jpn Acad., Ser. A 83, 148-151 (2007)

7. Hayman, WK: Meromorphic Functions. Oxford Mathematical Monographs. Clarendon, Oxford (1964)

8. Yang, L: Value Distribution Theory. Springer, Berlin (1993)

9. Yi, HX, Yang, CC: Uniqueness Theory of Meromorphic Functions. Science Press, Beijing (2003)

10. Laine, I: Nevanlinna Theory and Complex Differential Equations. de Gruyter, Berlin (1993)

11. Hayman, WK: On the characteristic of functions meromorphic in the plane and of their integrals. Proc. Lond. Math. Soc. 14, 93-128 (1965)

12. Bergweiler, W, Ishizaki, K, Yanagihara, N: Meromorphic solutions of some functional equations. Methods Appl. Anal. 5(3), 248-259 (1998). Correction: Methods Appl. Anal. 6(4), 617-618 (1999)

13. Gundersen, G, Heittokangas, J, Laine, I, Rieppo, J, Yang, D: Meromorphic solutions of generalized Schröder equations. Aequ. Math. 63, 110-135 (2002)

doi:10.1186/1029-242X-2013-331

Cite this article as: Zhang: On a question of Beardon. Journal of Inequalities and Applications 2013 2013:331. 